\title{
Clinical and radiographic observations from four patients with primary hepatic lymphoma
}

\author{
HUI-JUAN HU, YAN-JUAN QU, ZHI-XIONG TIAN and ZAI-PENG ZHANG \\ Department of Computed Tomography, Zhongnan Hospital of Wuhan University, Wuhan, Hubei 430071, P.R. China
}

Received July 3, 2014; Accepted February 23, 2015

DOI: $10.3892 /$ etm.2015.2940

\begin{abstract}
The aim of the present study was to delineate the radiographic and clinical features of primary hepatic lymphoma (PHL). Four histopathologically confirmed cases of PHL were analyzed with respect to the radiological, clinical and pathological characteristics. The main clinical manifestations included upper right quadrant pain and lymphoma-associated B symptoms, such as fever, night sweating and weight loss. All the patients had elevated serum levels of lactate dehydrogenase. Furthermore, all the patients underwent plain and enhanced computed tomography examinations, which identified low-density lesions without marked enhancement. Solitary masses were observed in two cases, while multiple focal lesions were noted in one case and diffuse multi-speckled nodules were observed in one case. Two patients underwent abdominal magnetic resonance imaging, which revealed lesions that were hyperintense on T1-weighted imaging (WI) scans and hypointense on T2WI scans, and exhibited slight to moderate enhancement with a dynamic contrast-enhanced protocol. In one case, vessels were visible within the lesion. Therefore, the present study concluded that PHL is a rare condition that exhibits non-specific clinical and radiological features. A combination of imaging results and clinical manifestations can be used to facilitate a diagnosis of PHL.
\end{abstract}

\section{Introduction}

Non-Hodgkin lymphoma (NHL) is a common lymphoproliferative disease. Liver involvement, which defines an advanced classification of the disease, occurs in $10 \%$ of patients with NHL (1).

Although the liver contains lymphoid tissue, host factors may render the liver inhospitable for the development of

Correspondence to: Professor Yan-Juan Qu, Department of Computed Tomography, Zhongnan Hospital of Wuhan University, 169 East Lake Road, Wuhan, Hubei 430071, P.R. China

E-mail: hjcndoc@126.com

Key words: hepatic lymphoma, non-Hodgkin lymphoma, computed tomography, magnetic resonance imaging a malignant lymphoma (2). Accordingly, primary hepatic lymphoma (PHL) is a very rare type of malignancy, accounting for $\sim 0.016 \%$ of all NHL cases (3). The most common symptoms of PHL at presentation are abdominal pain and general malaise. Additional presentation symptoms may include B symptoms, which encompass low-grade fever, fatigue, night sweats and weight loss. In addition, a mass with or without jaundice can occur, and in exceptional cases, fulminant hepatic failure has been observed (4-7). The majority of cases of PHL derive from B-cell lymphoma (88.6\%) and histologically diffuse large B-cell lymphoma (52.5\%), whereas other histological types account for $<5 \%$ of PHL cases $(4,8,9)$. The pathogenesis of PHL is yet to be fully established, although several possible factors, including viral infection, cirrhosis and immunosuppressive drugs, have been implicated $(2,3)$. Due to the infrequency of PHL occurrence, the clinical and radiological features of PHL have not been fully identified. Accordingly, PHL is very difficult to diagnose accurately and is often misdiagnosed as a hepatocellular carcinoma (HCC), metastatic tumor or liver abscess. Therefore, the aim of the current study was to report the clinical, radiological and histopathological analysis observations of four PHL cases, and briefly review the literature.

\section{Materials and methods}

Patient selection and data collection. A search of the medical records of patients diagnosed with histopathologically confirmed PHL between October 2007 and May 2013 was conducted. Ultimately, four cases with radiological evidence and a pathological diagnosis of PHL were included in the study. All patient information concerning the demographic data, clinical and laboratory presentations, underlying diseases, imaging manifestations and pathological results were recorded. The study was conducted in accordance with the Declaration of Helsinki and with approval from the Ethics Committee of Zhongnan Hospital of Wuhan University (Wuhan, China). Written informed consent was obtained from all the participants.

Imaging technique. With the patient in the supine position, plain and two-phase (arterial and portal vein phases) iodinated contrast-enhanced computed tomography (CT) scans were obtained in a craniocaudal direction using either of two scanners, namely the Sensation 16 CT or Somatom Definition 
dual-source CT (Siemens Medical Solutions, Erlangen, Germany). Routine scanning was conducted at an 8-mm section thickness and a 5-mm scan increment; scans were reconstructed with a 2-mm thickness using an appropriate algorithm. A dual-syringe injector system (Medrad Medical Equipment Trading Co., Ltd., Beijing, China) was used to intravenously administer $100 \mathrm{ml}$ non-ionic contrast media (Ultravist; $370 \mathrm{mgI} / \mathrm{ml}$; Bayer AG, Leverkusen, Germany) at a rate of $3 \mathrm{ml} / \mathrm{sec}$, followed by a $20-30-\mathrm{ml}$ saline chaser bolus. Magnetic resonance imaging (MRI) scans were acquired using a Magnetom Trio 3.0T scanner (Siemens Medical Solutions). Routine scanning of transverse sections was performed with $\mathrm{T} 2$-weighted fast spin-echo sequences, two-dimensional gradient echo in the axial plane, and T2-weighted half-Fourier acquisition single-shot turbo spin echo without fat saturation. A three-dimensional gradient echo sequence (VIBE) with fat saturation was performed prior to and following the intravenous bolus administration of gadopentetate dimeglumine (Magnevist; Schering, Berlin, Germany) at a dose of $0.1 \mathrm{mmol} / \mathrm{kg}$.

Retrospective imaging review. Two radiologists with $>10$ years experience evaluated the imaging features, including the location, shape, margination, homogeneity/heterogeneity, density, signal intensity and enhancement pattern.

Histopathological review. One patient underwent a surgical resection and three patients underwent an ultrasound-guided biopsy. All liver samples were fixed in $10 \%$ neutral-buffered formalin and processed routinely for paraffin embedding, followed by sectioning $(4 \mu \mathrm{m})$ and staining with hematoxylin and eosin. An immunohistochemical study was performed in three patients.

\section{Results}

Clinical characteristics. All four patients were male, with ages ranging between 38 and 64 years (average age, 56 years). Their basic information and clinical characteristics are summarized in Table I. All four patients complained of right upper abdominal pain and bloating, belching, discomfort, fatigue and weight loss, among other symptoms, and all exhibited elevated serum lactate dehydrogenase (LDH) levels. Two patients had abnormal liver function test results, and one patient exhibited an elevated $\beta_{2}$-microglobulin level. The serum levels of $\alpha$-fetoprotein (AFP), carcinoembryonic antigen (CEA) and other tumor markers were normal. In addition, the serology results were negative for human immunodeficiency virus (HIV) and the hepatitis B and $\mathrm{C}$ viruses (HBV and $\mathrm{HCV}$; Table I). All patients underwent plain CT, enhanced CT and T1-weighted imaging (Figs. 1-4), and three patients underwent immunohistochemistry.

Imaging features. The four patients underwent plain and enhanced CT evaluations. From the scans, one lesion was identified in the right lobe of the liver, one lesion was located in the quadrate lobe, while two lesions were located in the right and left lobes. One patient presented with diffuse, multi-speckled lesions (Fig. 1A), two patients presented with solitary masses (Figs. 2A and 3A), wheras the final patient presented with multiple nodules and masses (Fig. 4A).

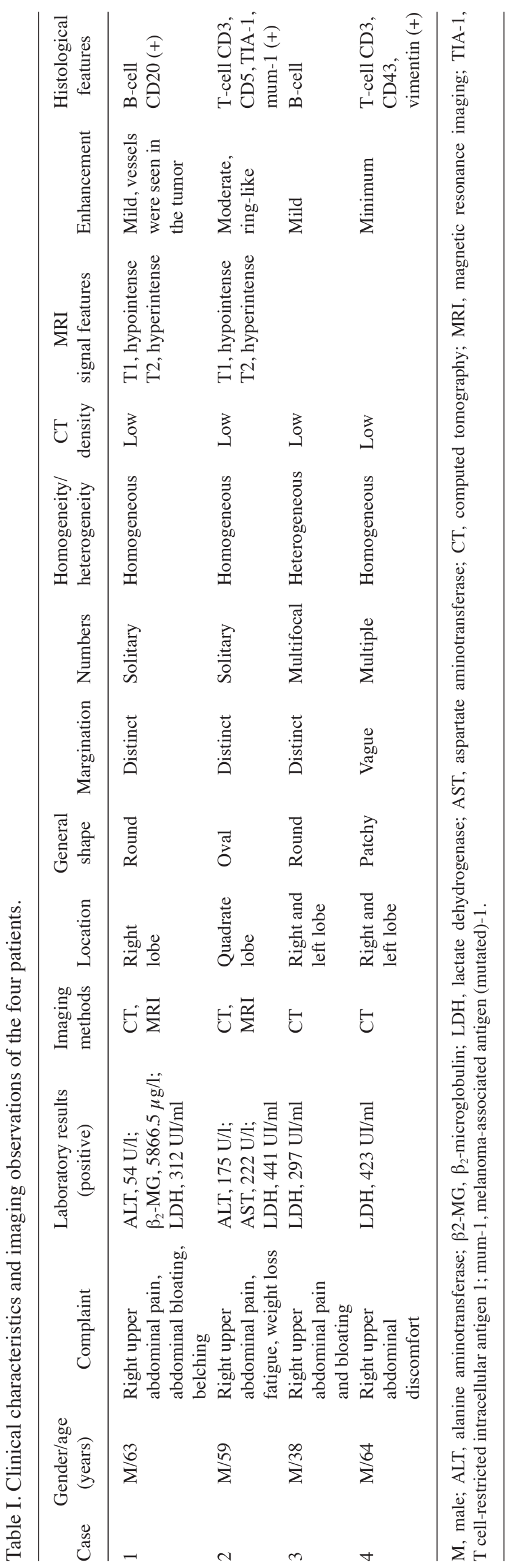


A

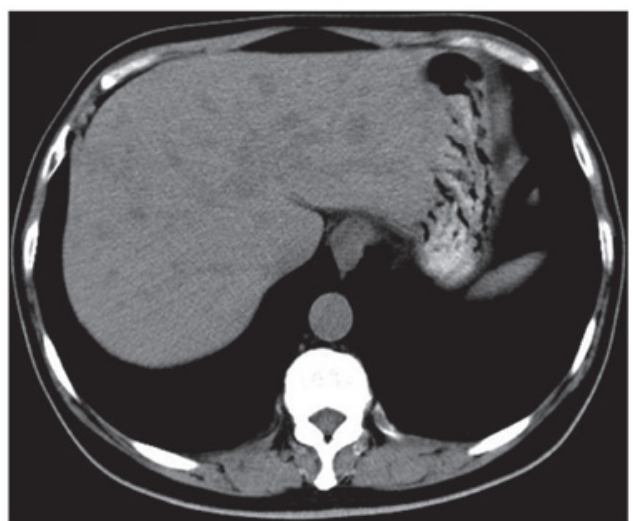

B

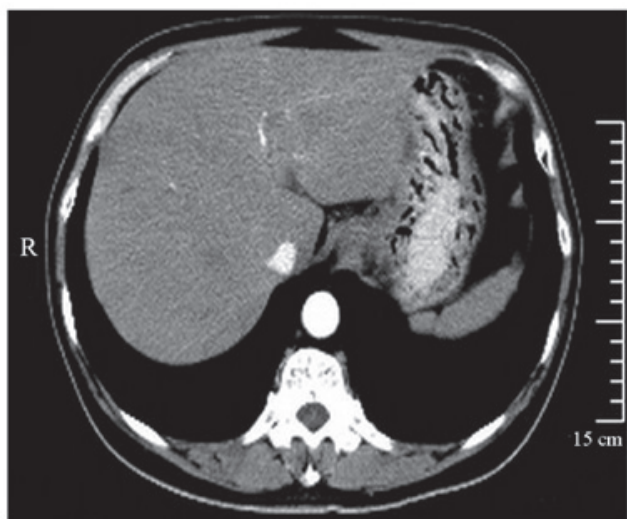

Figure 1. A 64-year-old male was admitted to hospital with right upper abdominal discomfort. (A) Transverse plain computed tomography (CT) image shows multi-speckled hypodense lesions involving the right and left lobe. (B) On the enhanced CT image, the lesions show mild enhancement
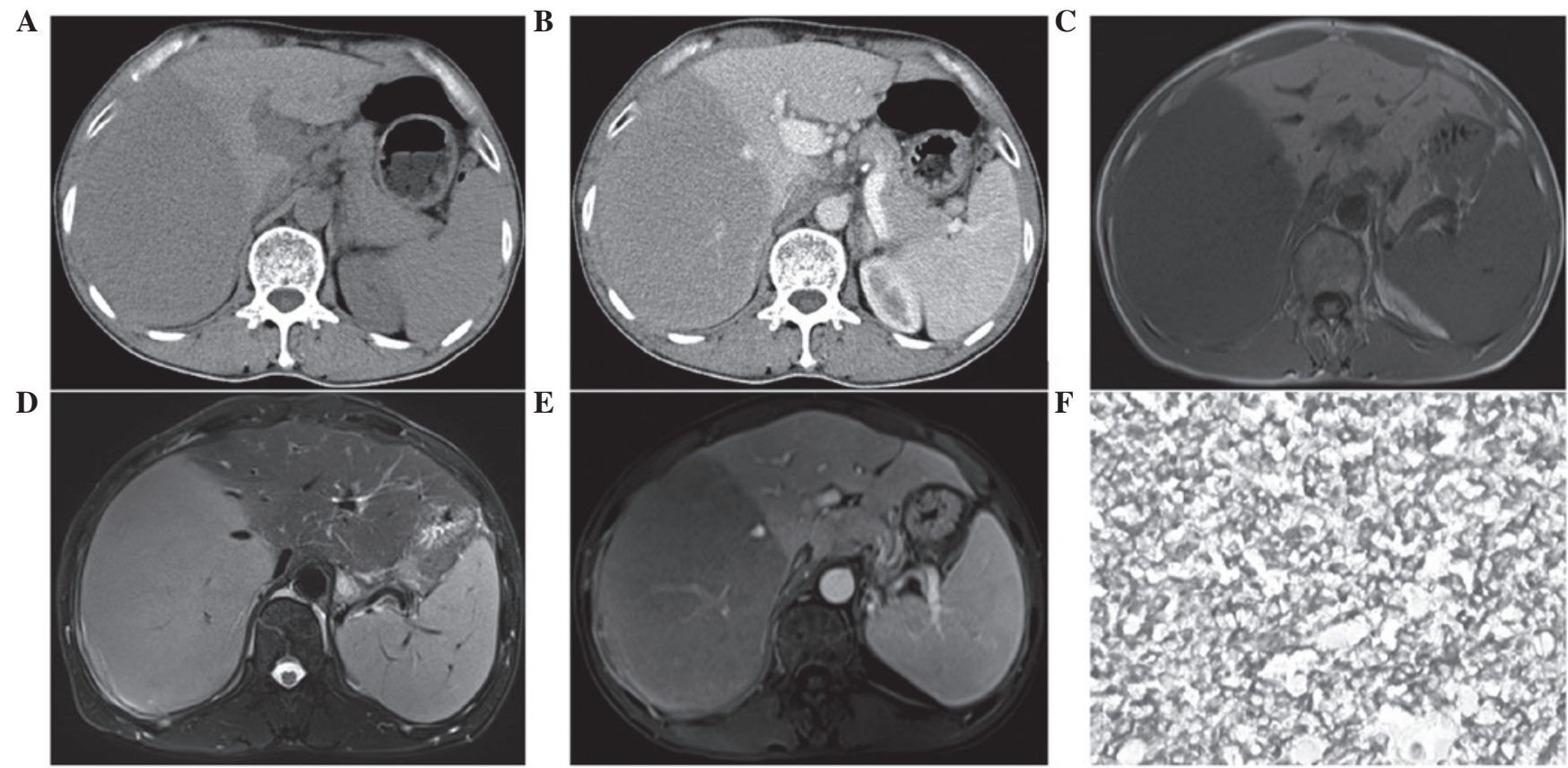

Figure 2. A 63-year-old man was admitted to hospital with symptoms of right upper abdominal pain, bloating and belching. (A) Transverse non-enhanced computed tomography (CT) image shows a homogeneous and low-density mass located in the right lobe of the liver. (B) On the contrast-enhanced CT image, the lesion shows mild enhancement and the blood vessels can be observed without any deformity. (C) The lesion has a low signal intensity on the transverse T1-weighted image. (D) The lesion has a high signal intensity on the transverse T2-weighted image. (E) On the post-contrast T1-weighted image, the lesion shows a mild and homogeneous enhancement, and enhanced liver vessels can be observed in the mass. (F) Immunohistochemistry analysis of the specimen shows positive staining of the cells for CD20 (magnification, $\mathrm{x} 400$ ).

CT images were available for all four patients. All the lesions appeared as low-density areas on the non-enhanced CT scans. Three of the lesions were homogeneous and one lesion was heterogeneous with a necrotic area in the center. On the contrast-enhanced CT scans, two patients exhibited minimal or no enhancement (Figs. 1B and 2B), one patient exhibited a moderate ring-like reinforcement (Fig. 3B), and one patient exhibited mild, uneven enhancement (Fig. 4B). In one lesion, normal blood vessels were visible (Fig. 2B).

Two patients underwent MRI evaluations with gadopentetate dimeglumine-based contrast enhancement. In these images, two round masses with distinct edges were observed. On the T1-weighted imaging (WI) scans, all the lesions appeared hypointense. On the T2WI scans, all the lesions were moderately hyperintense. Vessel signals were observed in one patient (Fig. 2C and D). On the post-contrast T1WI, one lesion exhibited a mild and homogeneous pattern, in which the enhanced liver vessels were visible (Fig. 2E), whereas another patient exhibited a moderate and ring-like pattern of enhancement (Fig. 3C).

Pathological observations. Histopathological specimens were obtained from the four patients via an ultrasound-guided biopsy or surgery. The pathological sections showed diffuse lymphocytic infiltration in the tissues (Fig. 4C). All the patients were pathologically confirmed with a diagnosis of PHL. Furthermore, two patients were classified as having the B-cell type, while the remaining two patients were classified with the T-cell type. Three patients underwent immunohistochemical examinations, revealing positivity for B-cell or T-cell 
A

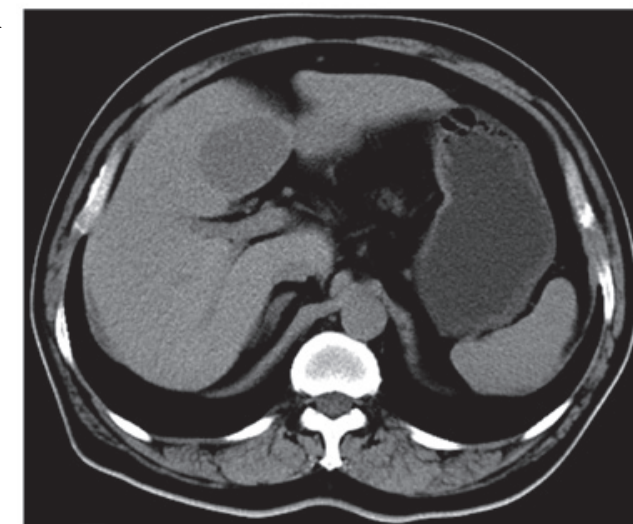

C

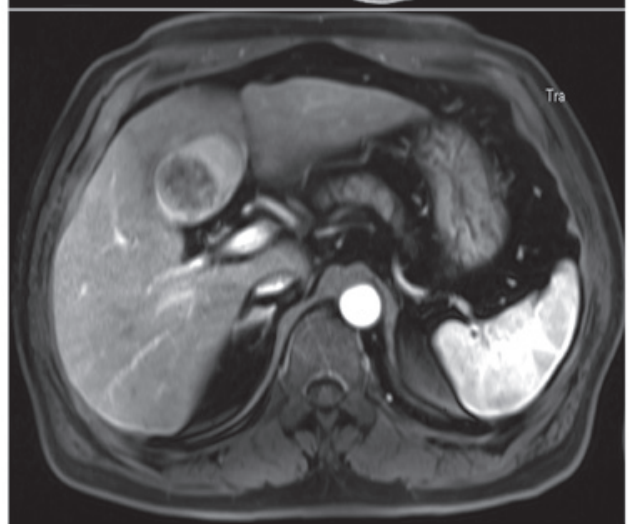

B

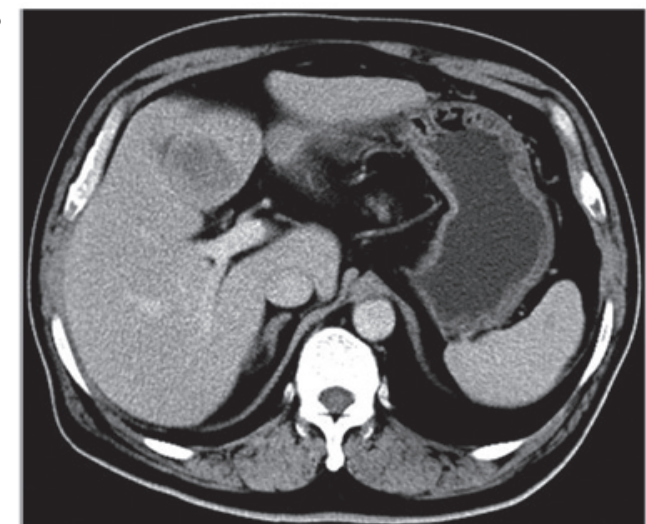

D

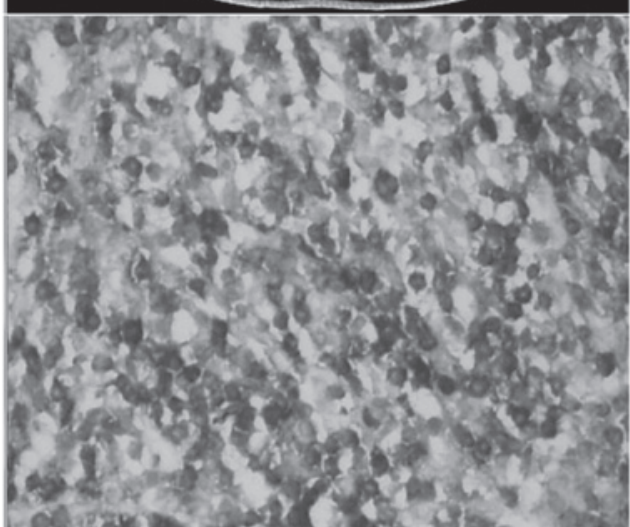

Figure 3. A 59-year-old male was admitted to hospital with symptoms of right upper abdominal pain, fatigue and weight loss. (A) Transverse non-enhanced computed tomography (CT) image shows a low-density mass with a distinct boundary, located in the quadrate lobe of the liver. (B) Portal phase enhanced CT image shows slight enhancement in the peripheral of the lesion. (C) On the post-contrast T1-weighted image, the lesion shows a moderate and peripheral enhancement. (D) Immunohistochemistry of the specimen revealed positive staining of the cells for CD3 (magnification, $\mathrm{x} 400$ ).
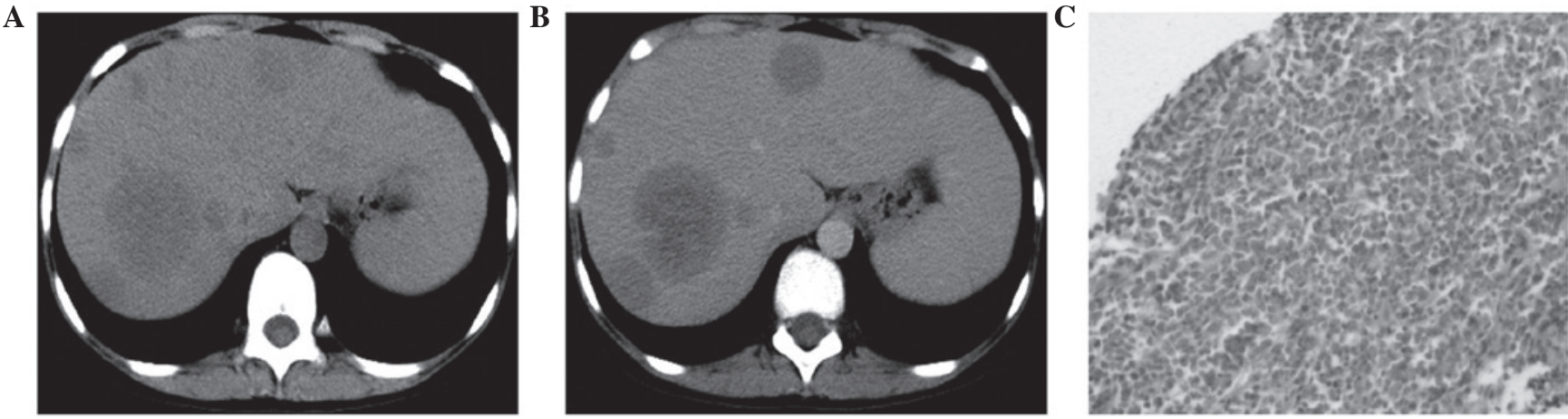

Figure 4. A 38-year-old male was admitted to hospital with right upper abdominal pain and bloating. (A) Transverse plain computed tomography (CT) image shows multifocal heterogeneous and ill-defined lesions that involve the right and left lobe. (B) On the enhanced CT image, the lesions show mild and heterogeneous enhancement. (C) Photomicrograph (hematoxylin and eosin; magnification, x40) reveals diffuse infiltrate with medium-to-large-sized lymphoid cells, which is suggestive of lymphoma.

markers, such as CD20 (Fig. 2F), CD3 (Fig. 3D), CD43, CD5, $\mathrm{T}$ cell-restricted intracellular antigen 1 and melanoma-associated antigen (mutated)-1 (Table I).

\section{Discussion}

According to the criteria outlined by Caccamo et al, PHL is defined as a lymphoma with only liver involvement at presentation, while there is absence of spleen, lymph node, peripheral blood, bone marrow or other tissue involvement for at least 6 months post-diagnosis (5). Although the liver contains lymphoid tissue, host factors can render the liver inhospitable for the development of a malignant lymphoma. Thus, PHL is a rare condition (2), accounting for $\sim 0.016 \%$ of all NHL cases (3).

The pathogenesis of PHL is yet to be fully established, although several possible etiological factors have been proposed. For example, there is evidence of an association between $\mathrm{HCV}$ infection and PHL. Previous studies have reported HCV infection rates ranging between 20 and $60 \%$ among patients with PHL, and this frequent association indicates that $\mathrm{HCV}$ may play a role in the pathogenesis of PHL $(10,11)$. However, 
a number of conflicting theories exist with regard to the association between HBV infection and PHL. Aozasa et al (12) reported a $20 \% \mathrm{HBV}$ surface antigen positivity rate in a series of 69 patients with PHL, whereas Yang et al (3) reported a $33.3 \% \mathrm{HBV}$ positivity rate in a series of nine patients with PHL. However, Noronha et al (4) concluded that any association between PHL and HBV was coincidental. PHL has also been associated with several other viral infections, including HIV and Epstein-Barr virus (EBV), inflammatory diseases, such as liver and primary biliary cirrhosis, and immunological disorders, including autoimmune diseases and immunosuppressive therapy $(13,14)$. However, none of the patients in the present study were found to have HCV infection or presented with signs of immunodeficiency, as determined by the negative serology results for active infection with HIV, HBV, HCV and EBV. Therefore, PHL is speculated to occur in patients without any liver disease.

Although PHL can occur at any age, the disease is more frequently observed in the fifth or sixth decade of life, with a male/female incidence ratio of 2-3/1 (15). In the present study, the majority of patients were elderly males, in accordance with the previously reported trend. The limited experience during the study revealed that PHL exhibited non-specific clinical manifestations. The most common symptoms of PHL at presentation are abdominal pain (39-70\%) and general malaise (4), along with additional symptoms, such as low-grade fever, night sweats and weight loss (more commonly known as B symptoms), nausea, vomiting and itching, as observed in the four patients of the current study. Liver function test results are abnormal in up to $70 \%$ of cases, with increased levels of LDH observed in $30-80 \%$ of cases, and increased expression levels of $\beta_{2}$-microglobulin, a well-described prognostic marker, observed in $90 \%$ of cases $(4,10,16,17)$. A previous study also reported that dynamic changes in the serum $\mathrm{LDH}$ level may serve as a diagnostic marker (14). However, the value of LDH as a diagnostic marker of PHL is limited due to the low level of specificity. The tumor markers, AFP and CEA, are present at normal levels in almost $100 \%$ of patients with PHL, which assists in the differential diagnosis (10). In the present study, all the patients had elevated serum LDH levels and normal AFP and CEA levels, whereas the alanine and aspartate aminotransferase levels were elevated in two patients and the $\beta_{2}$-microglobulin level was elevated in one patient.

The imaging features of hepatic lymphoma are non-specific. PHL can appear as a solitary lesion (39-60\%), as multiple lesions $(25-40 \%)$, or as a diffuse infiltration of the liver $(18,19)$. However, diffuse infiltration is rare and indicates a poor prognosis. Based on the imaging examinations of the four patients in the present study, two cases had solitary lesions, one patient had multi-speckled damage, and one case exhibited multiple nodular or massive lesions. On CT scans, PHL lesions appear as hypoattenuating lesions; half of these cases show no enhancement following intravenous administration of a contrast agent, whereas $\sim 25 \%$ of cases exhibit a ring of enhancement $(4,7,20)$. Classically, the MRI findings of PHL have been described as hypointense or isointense on T1WI and hyperintense on T2WI $(4,20)$. The imaging findings of the cases in the present study were similar to those reported in previous studies.

Considering the rarity of this disease entity, the non-specific clinical presentation and the non-specific laboratory and radiological features, confirming a diagnosis of PHL is very difficult. PHL can be confused with focal nodular hyperplasia, a primary hepatic tumor and hepatic metastases, among other conditions. Radiological and laboratory findings are extremely useful in aiding the differentiation between PHL and other diseases. In $80 \%$ of cases, HCC develops in the cirrhotic liver; these patients are $\mathrm{HBV}$ - or $\mathrm{HCV}$-positive and often have elevated AFP levels. In addition, HCC usually presents as a hypervascular tumor with marked enhancement in the arterial phase and washout in the delayed phase (21). Hepatic metastases are generally detected in patients with a history of a primary tumor. Furthermore, focal nodular hyperplasia usually appears as a hypointense or isodense lesion relative to the surrounding liver on CT images, and as an isointense lesion on MRI scans. These lesions are fairly homogeneous unless a central scar is present, which typically appears as a hypodense lesion on CT scans and bright on T2WI. This central scar, when present, is highly specific. In multiphasic CT or MRI studies, focal nodular hyperplasia typically exhibits rapid homogeneous contrast uptake in the early arterial phase and a rapid return to near-normal enhancement in the portal venous and delayed phases; however, the central scar may be slightly enhanced during the delayed phase due to the uptake of the contrast material by the fibroconnective tissue (22-25). By contrast, PHL appears as a low-density lesion on CT scans and exhibits no enhancement or minimally patchy or ring-like enhancement on contrast-enhanced CT. In the present study, the patients exhibited normal levels of AFP and other tumor markers, and exhibited hypointensity on T1WI scans and hyperintensity on T2WI scans. This combination of clinical and laboratory features allows a speculative diagnosis of PHL. However, a definite diagnosis requires histological results obtained via a liver biopsy or surgical resection, and the absence of lymphoproliferative disease outside the liver. The patients in the present study underwent a surgical resection or liver biopsy, and immunohistochemical staining of the liver biopsy samples with specific antibodies confirmed the diagnosis of PHL.

Limitations of the present study included the design as a retrospective study of only four cases, which relied primarily on a review of the medical records. Therefore, future studies should include a larger sample size, which may aid the comprehension of the radiological and clinical spectra of this disease presentation.

In conclusion, PHL is a notably rare disease that lacks specific imaging findings, clinical manifestations and biochemical markers, subsequently rendering the diagnosis of the condition very difficult. A biopsy or surgical resection should be performed in such cases, since only histological examination can ensure an accurate differential diagnosis. The presentation of clinical lymphoma B symptoms, an elevated serum LDH level, or minimal/rim enhancement on contrast CT or MRI scans, particularly in the absence of vessels with architectural distortion in the lesion, greatly supports a diagnosis of PHL.

\section{Acknowledgements}

The authors thank Zhi-gao Xu for her assistance in obtaining and reviewing the pathological sections. The present study was supported by the Natural Science Foundation of Hubei Province (grant no. 2012FFB04422). 


\section{References}

1. Agmon-Levin N, Berger I, Shtalrid M, Schlanger H and Sthoeger ZM: Primary hepatic lymphoma: a case report and review of the literature. Age Ageing 33: 637-640, 2004.

2. Yu YD, Kim DS, Byun GY, et al: Primary hepatic marginal zone B cell lymphoma: A case report and review of the literature. Indian J Surg 75 (Suppl 1): 331-336, 2013.

3. Yang XW, Tan WF, Yu WL, Shi S, Wang Y, Zhang YL, Zhang YJ and $\mathrm{Wu} \mathrm{MC}$ : Diagnosis and surgical treatment of primary hepatic lymphoma. World J Gastroenterol 16: 6016-6019, 2010.

4. Noronha V, Shafi NQ, Obando JA and Kummar S: Primary non-Hodgkin's lymphoma of the liver. Crit Rev Oncol Hematol 53: 199-207, 2005.

5. Caccamo D, Pervez NK and Marchevsky A: Primary lymphoma of the liver in the acquired immunodeficiency syndrome. Arch Pathol Lab Med 110: 553-555, 1986.

6. Cameron AM, Truty J, Truell J, et al: Fulminant hepatic failure from primary hepatic lymphoma: successful treatment with orthotopic liver transplantation and chemotherapy. Transplantation 80: 993-996, 2005.

7. Elsayes KM, Menias CO, Willatt JM, Pandya A, Wiggins M and Platt J: Primary hepatic lymphoma: imaging findings. J Med Imaging Radiat Oncol 53: 373-379, 2009.

8. Gomyo H, Kagami Y, Kato H, et al: Primary hepatic follicular lymphoma: a case report and discussion of chemotherapy and favorable outcomes. J Clin Exp Hematop 47: 73-77, 2007.

9. Takeuchi $\mathrm{N}$ and Naba K: Primary hepatic lymphoma is difficult to discriminate from a liver abscess. Case Rep Gastrointest Med 2014 925307, 2014

10. Page RD, Romaguera JE, Osborne B, et al: Primary hepatic lymphoma: favorable outcome after combination chemotherapy. Cancer 92: 2023-2029, 2001.

11. Bronowicki JP, Bineau C, Feugier P, et al: Primary lymphoma of the liver: clinical pathological features and relationship with HCV infection in French patients. Hepatology 37: 781-787, 2003.

12. Aozasa K, Mishima K and Ohsawa M: Primary malignant lymphoma of the liver. Leuk Lymphoma 10: 353-357, 1993.

13. Santos ES, Raez LE, Salvatierra J, Morgensztern D, Shanmugan N and Neff GW: Primary hepatic non-Hodgkin's lymphomas: case report and review of the literature. Am J Gastroenterol 98 2789-2793, 2003
14. Masood A, Kairouz S, Hudhud KH, Hegazi AZ, Banu A and Gupta NC: Primary non-Hodgkin lymphoma of liver. Curr Oncol 16: 74-77, 2009.

15. Haider FS, Smith R, Khan S and Rahman O: Primary hepatic lymphoma presenting as fulminant hepatic failure with hyperferritinemia: a case report. J Med Case Reports 2: 279, 2008.

16. Lei KI: Primary non-Hodgkin's lymphoma of the liver. Leuk Lymphoma 29: 293-299, 1998.

17. Avlonitis VS and Linos D: Primary hepatic lymphoma: a review. Eur J Surg 165: 725-729, 1999

18. Levy AD: Malignant liver tumors. Clin Liver Dis 6: 147-164, 2002 .

19. Cerban R, Gheorghe L, Becheanu G, Serban V and Gheorghe C: Primary focal T-cell lymphoma of the liver: a case report and review of the literature. J Gastrointestin Liver Dis 21: 213-216, 2012.

20. Maher MM, McDermott SR, Fenlon HM, et al: Imaging of primary non-Hodgkin's lymphoma of the liver. Clin Radiol 56: 295-301, 2001.

21. Hussain SM, Semelka RC and Mitchell DG: MR imaging of hepatocellular carcinoma. Magn Reson Imaging Clin N Am 10: 31-52, 2002.

22. Mortelé KJ, Praet M, Van Vlierberghe H, Kunnen M and Ros PR: CT and MR imaging findings in focal nodular hyperplasia of the liver: radiologic-pathologic correlation. AJR Am J Roentgenol 175: 687-692, 2000.

23. Terkivatan T, van den Bos IC, Hussain SM, Wielopolski PA, de Man RA and IJzermans JN: Focal nodular hyperplasia: lesion characteristics on state-of-the-art MRI including dynamic gadolinium- enhanced and superparamagnetic iron-oxide-uptake sequences in a prospective study. J Magn Reson Imaging 24: 864-872, 2006.

24. Hussain SM, Terkivatan T, Zondervan PE, Lanjouw E, de Rave S, Ijzermans JN and de Man RA: Focal nodular hyperplasia: findings at state-of-the-art MR imaging, US, CT and pathologic analysis. Radiographics 24: 3-19, 2004.

25. Blachar A, Federle MP, Ferris JV, et al: Radiologists performance in the diagnosis of liver tumors with central scars by using specific CT criteria. Radiology 223: 532-539, 2002 . 